$11 \mid 2007$

Varia

\title{
Hiérarchie, ordre et mobilité dans l'Occident médiéval (400-1100)
}

Auxerre, Centre d'études médiévales, 27-29 septembre 2006

\section{Geneviève Bührer-Thierry}

\section{(2) OpenEdition}

\section{Journals}

Édition électronique

URL : https://journals.openedition.org/cem/1062

DOI : $10.4000 /$ cem. 1062

ISSN : 1954-3093

Éditeur

Centre d'études médiévales Saint-Germain d'Auxerre

Édition imprimée

Date de publication : 15 août 2007

ISSN : 1623-5770

\section{Référence électronique}

Geneviève Bührer-Thierry, «Hiérarchie, ordre et mobilité dans l'Occident médiéval (400-1100) »,

Bulletin du centre d'études médiévales d'Auxerre | BUCEMA [En ligne], 11 | 2007, mis en ligne le 28 août 2007, consulté le 22 septembre 2022. URL : http://journals.openedition.org/cem/1062 ; DOI : https:// doi.org/10.4000/cem. 1062

Ce document a été généré automatiquement le 22 septembre 2022.

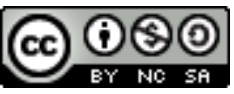

Creative Commons - Attribution - Pas d'Utilisation Commerciale - Partage dans les Mêmes Conditions 4.0 International - CC BY-NC-SA 4.0

https://creativecommons.org/licenses/by-nc-sa/4.0/ 


\section{Hiérarchie, ordre et mobilité dans l'Occident médiéval (400-1100)}

Auxerre, Centre d'études médiévales, 27-29 septembre 2006

\section{Geneviève Bührer-Thierry}

1 Les journées qui nous ont réunis à Auxerre du 27 au 29 septembre 2006 avaient pour objectif de réfléchir sur la notion de hiérarchie appliquée aux sociétés du haut Moyen Âge occidental qui, contrairement aux sociétés orientales de la même époque, ne semblent pas caractérisées par une hiérarchisation systématique. Certes, il ne s'agit nullement de sociétés égalitaires, mais de sociétés où les groupements horizontaux occupent autant de place - sinon plus - que les groupes fondés sur des liens verticaux. Il faut aussi souligner que dans ces sociétés «barbares ", l'idée même de la hiérarchie ne se réfère pas à une cascade de dignités et de fonctions distribuées par l'État, comme c'était le cas dans le monde romain du Bas-Empire et comme on le retrouve dans tout l'appareil d'État byzantin. On est d'ailleurs frappé de constater que seuls Céline Martin et Stefano Gasparri, c'est-à-dire les spécialistes des régions directement héritières du monde romain, l'Espagne wisigothique et l'Italie ostrogothique, ont cherché à pénétrer le sens et la pratique de la hiérarchie dans des sociétés dont l'idéologie se réfère encore - et plus qu'ailleurs - au système mis en place par les empereurs de l'Antiquité tardive. Si on excepte également Jean-Michel Picard qui raisonne à partir de sources irlandaises reflétant une façon bien particulière de concevoir le monde et la société, tous les autres participants ont choisi de parler de la période carolingienne où émerge une véritable réflexion, d'abord théorique, sur la hiérarchie, et où le pouvoir royal tente de " mettre en ordre » la société, notamment à partir du IXe siècle.

2 C'est en effet les écrits du monde carolingien qui nous donnent accès à l'essence et aux formes de la hiérarchie telles qu'on les conçoit en Occident. Plus que la nomenclature impériale héritée du Bas-Empire, c'est vers le modèle de la hiérarchie angélique que se tournent le plus volontiers les lettrés du monde carolingien lorsqu'ils cherchent un modèle de hiérarchie, et cela bien avant l'œuvre majeure de Jean Scot Erigène qui parachève cet édifice idéologique en tentant de réconcilier la hiérarchie terrestre et la hiérarchie céleste. 
3 La hiérarchie est donc d'abord d'origine divine, elle assigne à chaque homme sa place en ce monde, tout en préservant l'idée de l'unité de la communauté. Sur ce point, les communications de Rafaele SAVIGNY, Dominique IOGNA-PRAT et Alain RAUWEL montrent bien que cette hiérarchie ne s'oppose pas à la communauté, mais est au contraire ce qui la rend possible. En même temps, on peut penser avec HANS-WERNER GOETZ qu'une théorie comme celle des trois ordres repose avant tout sur le souci de mettre en garde les élites contre une surestimation d'elles-mêmes, au profit d'un discours qui promeut la dépendance des ordres hiérarchiques les uns envers les autres, au sein de la hiérarchie voulue par Dieu.

4 En outre, si l'origine divine de la hiérarchie permet à la communauté de se constituer comme une ecclesia, sans pour autant aboutir à une hiérocratie, elle permet aussi de hiérarchiser les sociétés entre elles, la société chrétienne répondant au modèle de la hiérarchie divine étant, de ce fait, la seule qui puisse être considérée comme authentiquement humaine.

5 Cet effort de conceptualisation propre à l'époque carolingienne s'est naturellement accompagné d'une volonté de mettre en place des formes de hiérarchie efficientes au sein même de la société : tout d'abord à travers l'expérience monastique, dont OttoGerhard oExLE montre qu'elle passe d'un idéal d'égalité dans la communauté à un idéal hiérarchique qui s'appuie sur le modèle clérical, dont la hiérarchie s'impose assez rapidement comme modèle de référence, tout en subissant elle-même de multiples modifications comme l'étudient Steffen PATZOLD, Alain RAUWEL et Hedwig RöcKELEIN.

Enfin reste le problème de l'articulation entre la pensée hiérarchique produite par les clercs visant à fournir le soubassement idéologique à l'œuvre de "mise en ordre » propre aux Carolingiens, et les formes perceptibles de la hiérarchie sociale qui obéissent aussi à d'autres critères qui fondent la distinction sociale.

7 Ces critères reflètent essentiellement les relations qu'un individu entretient avec l'ensemble de la société et d'abord avec lui-même : ainsi, tout en bas de la hiérarchie, on trouve ceux qui ne disposent même pas de leurs propres corps, signe systématiquement interprété comme celui de la non-liberté. Doivent être également prises en compte, les relations de l'individu à l'autorité légitime qui, la plupart du temps, visent à conforter sa position sociale, mais dont on observe aussi qu'elles sont absolument nécessaires à un maintien durable dans la hiérarchie, comme le soulignent pour l'Italie Laurent FELLER et Vito LORÉ. En outre, il ne s'agit pas seulement d'appartenir à une hiérarchie publique, mais aussi de jouer un rôle d'intermédiaire entre les communautés locales et le pouvoir «central» qui, peut-être, n'a pas forcément intérêt à soutenir les élites les plus puissantes mais peut aussi privilégier un partenariat avec des élites issues d'un milieu moins prestigieux mais, de ce fait, moins remuantes, comme le propose Thomas LIENHARD.

8 On ne s'étonnera pas qu'un des critères déterminants soit la relation qu'un individu entretient avec la personne du prince : la possibilité d'accéder à la faveur du prince et la proximité royale sont à la fois un moyen de renforcer le pouvoir des élites et un puissant ferment de mobilité sociale au sein de cette hiérarchie polymorphe. Philippe DEPREUX décrit la hiérarchie aulique qui fait écran entre le roi et ses sujets, même si cette hiérarchie conserve un caractère encore fluide et peu codifié, surtout si on la compare à ses homologues byzantins. On est néanmoins frappé de l'effort fait par les Carolingiens pour instaurer un lien quasiment juridique entre le roi et ses sujets grâce à 
la généralisation du serment de fidélité qui couronne l'ensemble de l'édifice, ainsi que le montre Stefan ESDERS.

9 Enfin, la hiérarchie se manifeste également au sein des relations «horizontales» comme les guildes, étudiées par Alban GaUTIER, qui souligne que même au sein de structures appelées à promouvoir l'égalité et la solidarité entre ses membres, peuvent se développer de nombreuses stratégies de distinction. Et Charles MÉRIAUX montre bien comment la volonté de renforcer les structures hiérarchiques au sein du clergé rural a dû s'appuyer sur la récupération de solidarités ecclésiastiques horizontales.

Ces dernières considérations nous ont permis de revenir au début de notre propos et de montrer finalement comment le discours, qui prône un modèle hiérarchique sans opposition à la communauté, soutient une réalité sociale où les liens verticaux ne se substituent pas aux liens horizontaux, mais cherchent à les englober dans une dynamique au service de la construction du projet global qui était celui des Carolingiens.

11 Rappel du programme :

\section{Hiérarchie : horizons d'une enquête collective}

- R. LE JAN, F. BOUGARD, Hiérarchie : le concept et son champ d'application dans les sociétés du haut Moyen Âge

-J.-M. PICARD, Christianisation et hiérarchisation de la société irlandaise des VIIe et VIIIe siècles

- R. SAVIGNI, La communitas christiana dans l'ecclésiologie carolingienne

- D. IOGNA-PRAT, Penser l'Église et la société après le Pseudo-Denys l'Aréopagite

-H. W. GOETZ, Les ordines dans la théorie médiévale de la société : un système hiérarchique ?

\section{Ordres et grades ecclésiastiques : liturgie, ecclésiologie et histoire sociale}

-A. RAUWEL, La hiérarchie interne à l'ordre sacerdotal : épiscopat et presbytérat des temps patristiques à Pierre Lombard

- ch. MÉRIAUX, Les clercs ruraux et la hiérarchisation de la société carolingienne : une première enquête dans la province de Reims

-S. GASPARRI, Reclutamento sociale e ruolo politico dei vescovi in Italia, secoli VI-VIII

-S. PATZOLD, Créer un grade ecclésiastique : métropolitains et archevêques du royaume franc (VIIIe-IXe siècles)

\section{Monachisme et hiérarchie}

• O. G. OEXLE, Mönchtum und Hierarchie im Okzident

-H. RÖCKELEIN, Hiérarchie et ordre dans le monachisme féminin

\section{Hiérarchie et société laïque}

- S. ESDERS, Fidélité et diversité juridique

- L. FELLER, Hiérarchies et mobilité sociale dans le monde rural (IXe-XIe siècles)

-V. LORÉ, Poteri pubblici ed elite rurali nell'Italia meridionale longobarda (Secoli IX-XI)

- Th. LIENHARD, L'empereur et les élites urbaines : Charlemagne face à Salzbourg et à Rome

- Ph. DEPREUX, Hiérarchie et ordre au sein du palais : l'accès au prince.

- C. MARTIN, Hiérarchie et service dans le monde wisigothique : la militia des laïcs

- C. LA RoccA, Distinguersi per la ricchezza da Venanzio Fortunato a Raterio

-A. GAUTIER, Discours égalitaire et pratiques hiérarchiques dans les ghildes anglo- saxonnes

Conclusions par G. BÜHRER-THIERRY 
INDEX

Mots-clés : hiérarchie 